\title{
Electron Microscopic Measurement of the Size of the Optical Focus in Laser Scanning Microscopy
}

\author{
Alison McDonald, ${ }^{1, *}$ William B. Amos, ${ }^{2}$ and Gail McConnell ${ }^{1}$ \\ ${ }^{1}$ Strathclyde Institute for Pharmacy and Biomedical Sciences, University of Strathclyde, 161 Cathedral Street, \\ Glasgow G4 ORE, UK \\ ${ }^{2}$ MRC Laboratory of Molecular Biology, Hills Road, Cambridge CB2 OQH, UK
}

\begin{abstract}
We describe a method for measuring the lateral focal spot size of a multiphoton laser scanning microscope (LSM) with unprecedented accuracy. A specimen consisting of an aluminum film deposited on a glass coverslip was brought into focus in a LSM and the laser intensity was then increased enough to perform nanoablation of the metal film. This process leaves a permanent trace of the raster path usually taken by the beam during the acquisition of an optical image. A scanning electron microscope (SEM) was then used to determine the nanoablated line width to high accuracy, from which the lateral spot size and hence resolution of the LSM can be determined. To demonstrate our method, we performed analysis of a multiphoton LSM at various infrared wavelengths, and we report measurements of optical lateral spot size with an accuracy of $20 \mathrm{~nm}$, limited only by the resolution of the SEM.
\end{abstract}

Key words: resolution, laser scanning microscopy, SEM, ablation

\section{INTRODUCTION}

The resolving power of a microscope is the most important feature of the optical system and influences the ability to distinguish between fine details of a specimen. According to the Abbe equation, modified by Rayleigh, the resolution (radius) $r$ is given by

$$
r=\frac{0.61 \lambda}{N . A .}
$$

where $\lambda$ is wavelength and N.A. is numerical aperture (Abbe, 1884; Ditchburn, 1991). This lateral resolution is particularly important in life and materials science research where submicron scale objects are frequently visualized using methods such as confocal and multiphoton laser scanning microscopy (Sheppard \& Kompfner, 1978; Amos \& White, 1987; Denk et al., 1990; Inoué, 2006).

The current gold-standard method for measuring the resolution of a standard laser scanning microscope (LSM) consists of imaging fluorescently labeled beads or prefabricated etched gratings on a slide and then analyzing the optical image (Oldenbourg et al., 1993; Cox \& Sheppard, 2004; Zucker, 2006). Clearly, the accuracy of this technique can never exceed the resolution of the objective lens used, and hence precise measurement of the lateral spatial resolution of a confocal or multiphoton LSM is limited to a few hundreds of nanometers, at best. One of the key problems in using fluorescent beads for resolution measurements of multiphoton LSMs arises from the use of high peak intensity lasers: even at very modest applied powers, the peak intensity of the radiation used for excitation exceeds the low

Received October 18, 2011; accepted February 21, 2012

*Corresponding author. E-mail: alison.mcdonald@strath.ac.uk damage threshold of the small diameter beads, and thus a resolution measurement cannot be performed. In superresolution optical microscopy, methods including stimulated emission depletion (STED) (Klar et al., 2000), stochastic processes (Betzig et al., 2006), and sequential photobleaching (Gordon et al., 2004) can be used to increase spatial resolution. However, these methods depend heavily on specialized photochemistry, software, and instrumentation, and cannot be used easily to measure the lateral spot size or resolution of standard multiphoton microscopes.

Away from microscopy, measurement of laser beam radii is usually performed using knife-edge methods (Siegman et al., 1998), but here the minimum resolvable radius is typically $>5 \mu \mathrm{m}$, simply because of the mechanical tolerances of the instrumentation used. To overcome this, to some extent, Liu (1982) reported a technique for more accurate measurement of stationary beam radii from a frequency-doubled and frequency-quadrupled 20 picosecond-pulsed Nd:YAG laser at wavelengths of $532 \mathrm{~nm}$ and $266 \mathrm{~nm}$. The laser source was focused onto single-crystal silicon surfaces, resulting in a microablation process, and a direct image of the distribution of the laser-energy density could be made using an optical microscope. From this image, and with simple Gaussian-beam analysis, the beam radius could be measured. However, although compatible with high repetition rate, ultra-short pulsed lasers (Liu et al., 1997; Tan et al., 2008), this method is limited to beam radii that are larger than the resolving power of the objective lens used to analyze the microablated spot. As such, this method has not been applied to measuring beams of submicron radius, such as those routinely used in laser scanning microscopy.

Clearly, the weakness of these beam radius measurement techniques is the optical microscope as the measuring device, and a higher resolution technique is needed for 
more accurate quantification. Our method is likely to prove useful for assessing laser focus quality because an optimized diffraction-limited focus is an essential prerequisite for STED (Hell \& Wichmann, 1994), structured illumination, and other methods that depend on a focused and scanned laser beam.

Here we describe a two-step technique to measure the lateral spatial spot size of a multiphoton LSM to unprecedented accuracy. The first step consisted of scanning an in-focus specimen consisting of a thin aluminum coating deposited on a glass substrate, using the raster scan parameters usually employed to acquire an image. Even at modest laser intensities, this nanoablation process left a permanent trace of the path of the scanning pulsed laser beam. The second step consisted of measuring the width of the ablated line in a scanning electron microscope (SEM). Analysis of the SEM image provided direct measurement of the nanoablated line width to an accuracy of $20 \mathrm{~nm}$, limited only by the resolution of the SEM, and from this measurement the spot size and resolution of the LSM could be derived to similar accuracy. We performed measurements at a range of wavelengths used in multiphoton microscopy and compare our results with theory, demonstrating the performance and accuracy of the technique. We note that our use of the SEM to throw light on the performance of optical microscopes has a precedent: a test plate produced by Norbert in 1885 proved, upon recent examination in an SEM, to have engraved lines $112.8 \mathrm{~nm}$ apart, beyond the resolution of the light microscope (Abbe, 1884; Turner \& Bradbury, 1966; Ditchburn, 1991).

\section{Materials and Method}

\section{Preparation of Aluminized Coverslips}

Aluminum was selected for this study because of its low melting point and hence low damage threshold, and also the ready visibility of a metallic specimen in the SEM. Circular glass coverslips $10 \mathrm{~mm}$ in diameter were cleaned in concentrated sodium hydroxide, washed in tap water and then distilled water, and dried before transfer to an Edwards coating unit equipped with standard rotary and diffusion pumps and a trap cooled with liquid nitrogen. A fragment of aluminum foil approximately $5 \mathrm{~mm}$ square was wound around a tungsten wire positioned approximately $15 \mathrm{~cm}$ above the coverslips. When a vacuum of at least $10^{-5}$ Torr was attained, current was passed through the tungsten wire, sufficient to heat it to a yellow glow, and the aluminum was evaporated onto the coverslips. A thickness monitor was not used, but coverslips were selected with a silvery appearance and an optical transmission of approximately $25 \%$ of daylight, there being variation in film thickness according to position of the coverslips within the evaporator. The selection process was performed to ensure that the coverslips chosen were aluminized and free of dirt or debris before imaging. After $24 \mathrm{~h}$, scratches were made on the specimen using a fine sharp needle. This was performed under a stereo microscope, and irregular scratches made. This served two purposes: (1) to help with focusing the aluminized coverslip on the LSM and (2) to assist with identifying nanoablated regions during SEM.

\section{Nanoablation Method}

Using the bright-field transmission imaging option available on the LSM, the aluminized coverslip specimen was brought into focus, using the scratches described above as a rough guide to focusing depth. In changing from the brightfield visible radiation to the near-infrared wavelength chosen for ablation, as was expected, the focal plane of the beam changed. To then focus the near-infrared radiation on the sample, the transmission photomultiplier (PMT) was used to adjust the focus for each wavelength. This was done using low intensity near-infrared radiation at low digital zoom (zoom factor $=1$ ) where the low intensity irradiation resulted in no alteration to the structure of the aluminized coverslip. In laser scanning microscopy, zooming of magnification is achieved by decreasing the line width and line spacing of the scanned raster, and the zoom factor is that by which the raster is scaled down relative to a standard raster size for a given objective lens. The same objective lens was used for both the bright-field transmission imaging and the laser scanning nanoablation, but with no digital zoom for the bright-field transmission but digital zoom (zoom factor $=4 \times$ ) applied during the nanoablation process, which increased the applied intensity to exceed the damage threshold of the aluminum specimen. With the sample in focus, a single frame was scanned, so that the laser beam passed only once over each scanned line.

To produce nanoablated lines on the aluminized coverslips, we used a standard multiphoton LSM system to raster scan and provide focusing of the laser spot. A schematic diagram showing the layout of the system is presented in Figure 1.

The LSM consisted of a scan head (Radiance 2000MP, Bio-Rad, Hercules, CA, USA) and an upright microscope (Eclipse E600FN, Nikon, Tokyo, Japan). The laser source for nanoablation was a free-space-coupled Ti:Sapphire laser (Chameleon, Coherent Inc., Santa Clara, CA, USA), which was wavelength tunable between $\lambda=720-900 \mathrm{~nm}$, with pulse durations of less than 200 femtoseconds across the tuning range at a repetition rate of $90 \mathrm{MHz}$. The laser beam was deflected by galvanometer scanning mirrors and then via a scan lens into a microscope, where the radiation was focused onto the specimen with a $20 \times / 0.75$ numerical aperture dry objective lens (Nikon, Plan Fluor). The scanning software (LaserSharp 2000, Bio-Rad) was used to control the scan speed, optical zoom (from $1 \times$ to $10 \times$ ), and the number of lines per scan. The average power at the sample plane was adjusted using an electro-optical modulator, which was controlled via the LaserSharp software. We used a $4 \times$ zoom and $64 \times 64$ line scan, with a pixel dwell time of $80 \mu \mathrm{s}$ for single frame irradiation.

This system provided sufficient pulse energies to exceed the empirically determined nanoablation threshold, as well as a low number of lines such that individual scan lines 


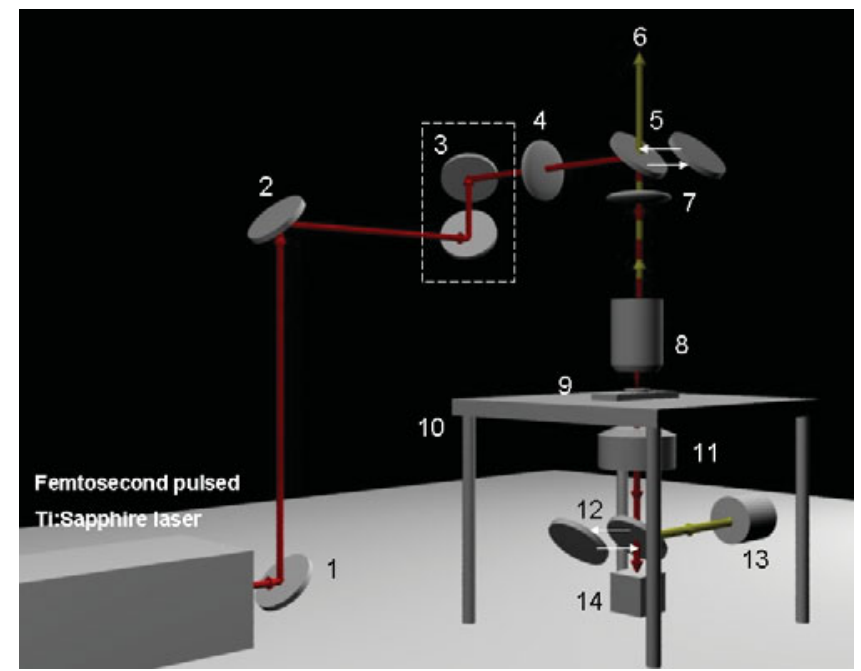

Figure 1. Laser scanning and bright-field microscope system. A $90 \mathrm{MHz}$ repetition rate, femtosecond-pulsed near-infrared emitting Ti:Sapphire laser (Chameleon, Coherent) was coupled using beam steering mirrors (1) and (2) into a LSM. This comprised a Bio-Rad Radiance system equipped with computer-controlled scanning galvo mirrors (3) and a scan lens (4) and an upright Nikon Eclipse E600FN microscope. A spectral reflector (5) was used to direct the near-infrared Ti:Sapphire laser radiation through the tube lens (7) toward the objective lens (8), which brought the laser light to a focus on a specimen (9) on a mechanical stage (10). Alternatively, the reflector could be moved aside, to allow incoherent white light to pass from a halogen lamp (13), to transilluminate the specimen and form an image in the eyepieces (6). The same $20 \times / 0.75$ numerical aperture dry objective lens was used in both bright field to bring the aluminized coverslip specimen into focus, and in the subsequent raster-scanning nanoablation experiments with the Ti:Sapphire laser. The position of the specimen was adjusted using the $X$ and $Y$ manual controls on the sample stage. For infrared transmission imaging, the light was collected by a substage condenser lens (11) and by displacing the mirror (12) directed toward a PMT detector (14). It was also possible to obtain infrared reflection images, using an epi-reflection detector (not shown).

were spaced far enough apart to be easily identified and consequently analyzed, although other configurations were possible. Given the $\mathrm{MHz}$ repetition rate of the laser used, we used the "N-on-one" accumulation model previously demonstrated in the ablation of metals (Jee et al., 1988). The dynamics of multipulse ablation are established, and for ablation of metals, the process is known to be cumulative and can be described by thermal stress-strain energy storage. In our experiment, the nanoablation process was a result of multiple pulses interacting with the substrate, on the order of 7,000 pulses per pixel, where the number of pulses was calculated using the pixel dwell time of the scan for the settings described previously.

To measure the beam radius with varying wavelength, a series of nanoablated lines scanned at varying power (and thus total pulse energy) was produced for six wavelengths across the tuning range of the Ti:Sapphire laser. The wavelengths selected were $\lambda=740,760,780,800,820$, and $850 \mathrm{~nm}$. The power transmission through the $20 \times / 0.75$
N.A. air immersion objective lens was measured before nanoablation experiments using a highly sensitive photodetector (NOVA II, Ophir Optronics Ltd, Jerusalem, Israel) for low power $(1-10 \mathrm{~mW})$ and a calorimetric power meter (407A, Newport Spectra-Physics, Oxfordshire, UK) for average power greater than $10 \mathrm{~mW}$. The average power was measured at the focus of the objective lens for each wavelength and for each power setting in the software (defined as $\%$ of laser power). The maximum average power used in this study was limited by the optical transmission of the LSM at each wavelength, and the range of total pulse energies examined was commensurate with the pulse energies commonly used in multiphoton imaging.

\section{SEM Method}

After nanoablation, the aluminized coverslip specimens were imaged using a SEM (JSM-6400, JEOL Ltd., Tokyo, Japan). The aluminum-coated glass coverslips were mounted on aluminum stubs using silver electro-dag paint (Agar Scientific, Stansted, Essex, UK). A low accelerating potential of $0.5-0.8 \mathrm{kV}$ was used throughout the study, first to minimize detrimental charging effects and also to provide high contrast images; however, this was at the cost of reduced resolution relative to high voltage SEM systems. The manufacturer quoted systematic resolution for this type of instrument is $3.5 \mathrm{~nm}$; however, our use of such low acceleration voltages gave a measured resolution of $20 \mathrm{~nm}$ or better. We therefore quote a resolution of $20 \mathrm{~nm}$ for the SEM images throughout this study, although we note that improved resolution may be possible with an alternative SEM system or, where possible, using higher accelerating potentials. Low magnification $(500-550 \times)$ and high magnification $(9,000 \times)$ SEM images were taken for analysis. The low magnification was used to locate the nanoablated regions, and the high magnification images were acquired for line-width analysis.

\section{Image Analysis}

Measurements of the nanoablated line widths in the high magnification SEM images were made by taking the $1 / e^{2}$ intensity profile of each line using ImageJ (National Institutes of Health, Bethesda, MD, USA). The $1 / e^{2}$ value was determined in accordance with Gaussian optics and as per the work of Liu (1982). For all images, $n=20$ intensity profiles were analyzed and an average nanoablated line width measured, from which the beam radius, spot size, and hence lateral resolution of the LSM could be calculated with an accuracy of $20 \mathrm{~nm}$, as described previously.

\section{Calculating the Optical Beam Radius from the Photoablated Line Width Data for Varying Total Pulse Energy}

For a Gaussian beam profile, the width of the nanoablated line $D$ is related to the incident beam radius $w_{0}$ by

$$
D^{2}=2 w_{0}^{2} \ln \left(\frac{E_{0}}{E_{t h}}\right)
$$



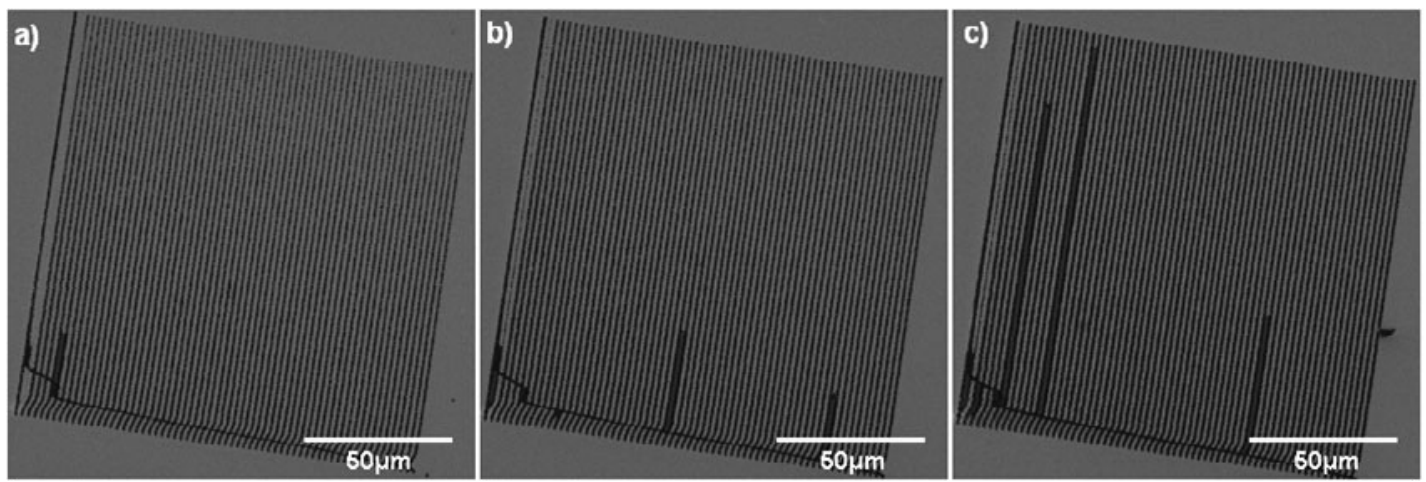

Figure 2. Single-frame nanoablated raster scans taken with varying total pulse energy (a) $1.5 \mu \mathrm{J}$, (b) $2.1 \mu \mathrm{J}$, and (c) $2.9 \mu \mathrm{J}$ applied to the specimen, at a fixed wavelength of $\lambda=760 \mathrm{~nm}$ were imaged using SEM at low magnification $(550 \times)$. The energies used correspond to applied average power of (a) $18 \mathrm{~mW}$, (b) $25 \mathrm{~mW}$, and (c) $34 \mathrm{~mW}$. The ablated lines were separated $2 \mu \mathrm{m}$ apart.
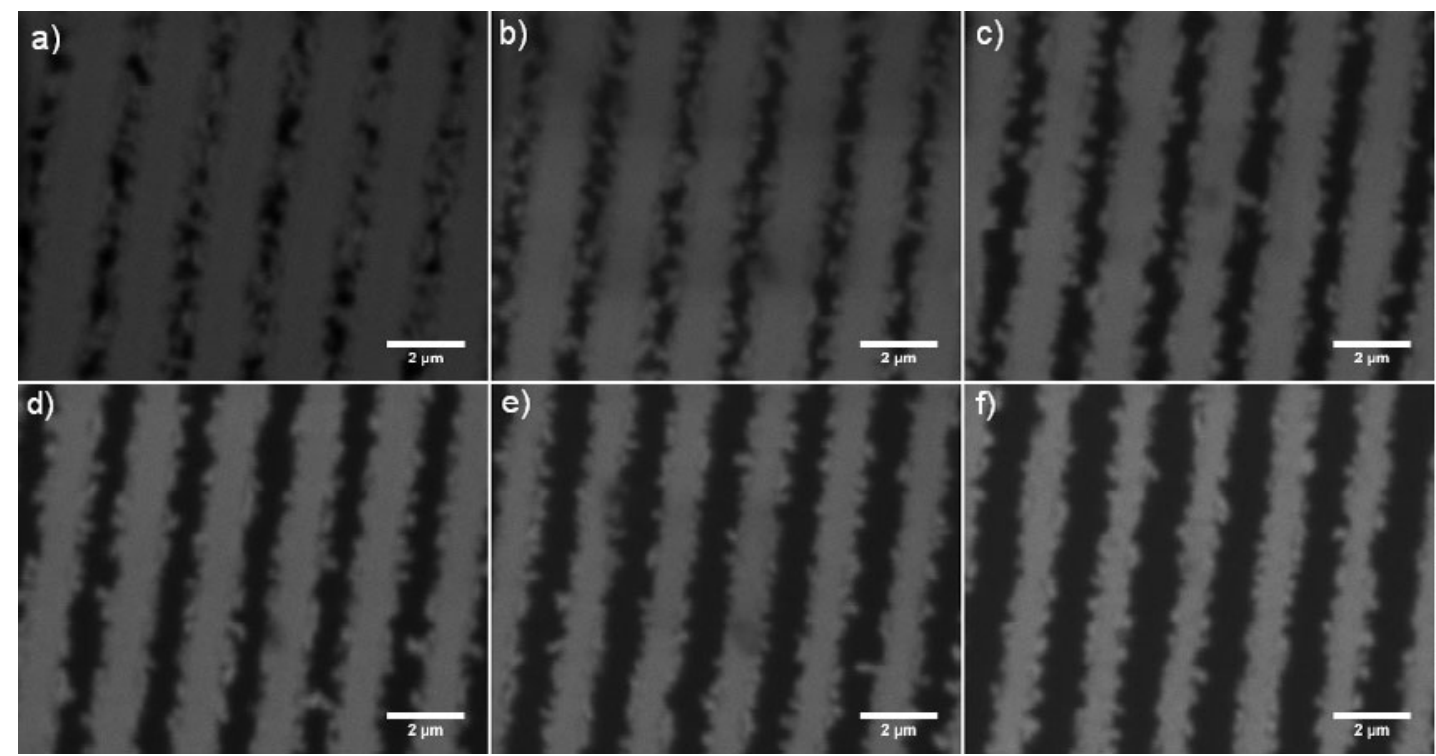

Figure 3. Single-frame nanoablated raster scans taken with varying total pulse energy (a) $0.7 \mu \mathrm{J}$, (b) $1.1 \mu \mathrm{J}$, (c) $1.5 \mu \mathrm{J}$, (d) $2.1 \mu \mathrm{J}$, (e) $2.9 \mu \mathrm{J}$, and (f) $4.3 \mu \mathrm{J}$ applied to the specimen, at a fixed wavelength of $\lambda=760 \mathrm{~nm}$ were imaged using SEM at high magnification $(9,000 \times)$. The energies used correspond to applied average power of (a) $8 \mathrm{~mW}$, (b) $13 \mathrm{~mW}$, (c) $18 \mathrm{~mW}$, (d) $25 \mathrm{~mW}$, (e) $34 \mathrm{~mW}$, and (f) $51 \mathrm{~mW}$.

where $E_{0}$ is the laser pulse energy and $E_{t h}$ is the damage threshold energy, which in our experiment was the energy threshold for the nanoablation process (Liu, 1982).

Using the law of logarithms $\ln (A / B)=\ln (A)-\ln (B)$, equation (2) becomes

$$
D^{2}=2 w_{0}^{2}\left(\ln E_{0}-\ln E_{t h}\right)
$$

which is equivalent to

$$
D^{2}=2 w_{0}^{2} \ln E_{0}-2 w_{0}^{2} \ln E_{t h} .
$$

Equation (4) is recognizable in the form of a straight line $y=m x+c$, where $y=D^{2}, x=\ln \left(E_{0}\right)$ and the gradient $m=$ $2 w_{0}^{2}$ and $y$-intercept $c=-2 w_{0}^{2} \ln E_{t h}$. The intercept is clearly related to the threshold energy for the ablation process.
A least-squares fitting method was applied to obtain the best fit with experimentally measured values. From the gradient of this line, the beam radius could then be determined, and this was taken to be equivalent to the lateral spot size of the LSM.

\section{Results}

\section{Scanning Electron Microscopy}

Using the method described, nanoablated raster patterns were produced and SEM images obtained, with a resolution of $20 \mathrm{~nm}$. Figure 2 shows a series of representative SEM images, with nanoablation performed at a fixed wavelength of $\lambda=760 \mathrm{~nm}$ with increasing applied average power (and thus increasing total pulse energy). This increase in line width with increasing applied pulse energy was expected 

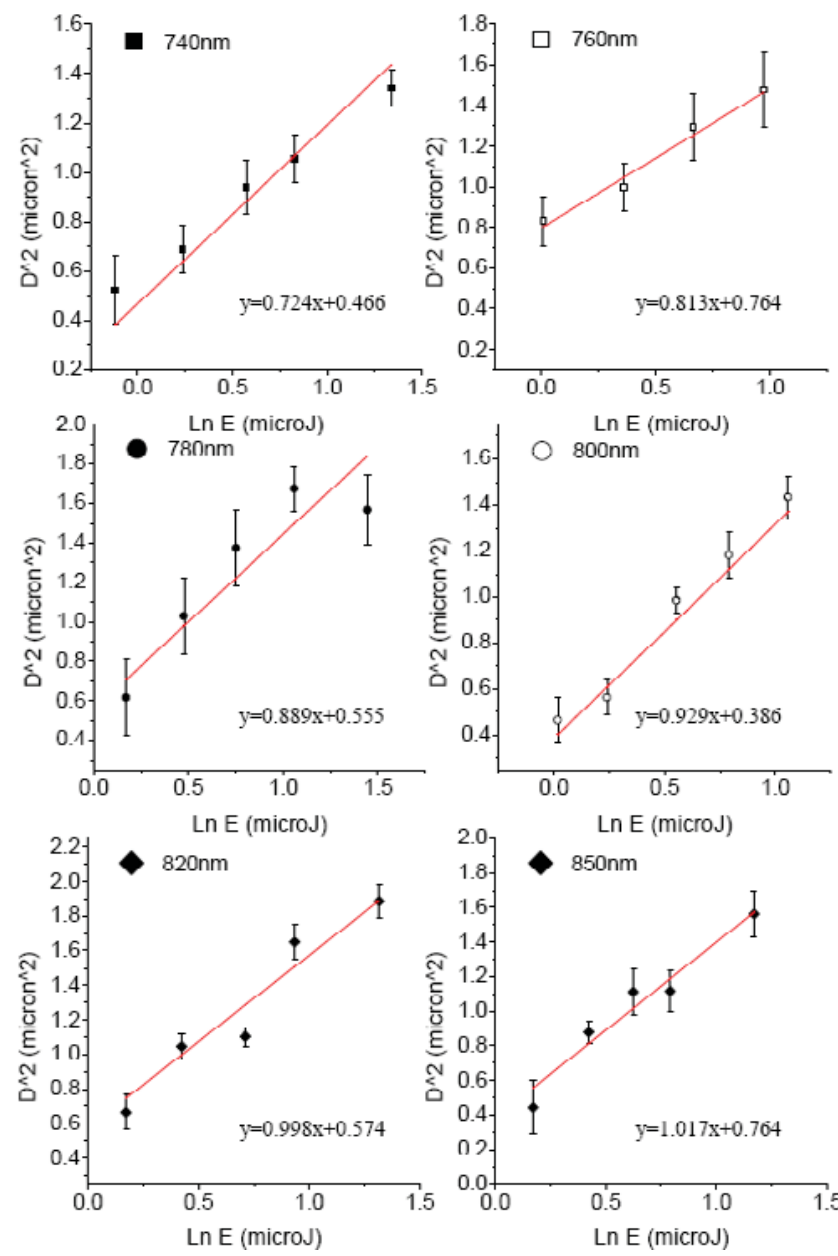

Figure 4. The square of the line widths obtained for each wavelength with increasing average power are plotted against the natural $\log$ of the total pulse energy employed in the nanoablation experiments. Mathematical fitting to experimental data was performed to obtain the best fit line and the gradient of the line, which, from Gaussian beam theory, was equal to $2 w_{0}^{2}$ and was used to calculate the beam radius and the spot size and hence to determine the lateral spatial resolution of the LSM. In these data, the error is the standard deviation in the mean.

because of the cumulative nature of the ablation process described previously, and with reference to the energy dependence of equation (2). The low magnification images show the overall condition of the nanoablated raster from which an area representative of the nanoablation process could be identified and a higher magnification image subsequently obtained. In each of these low magnification images, the beam flyback line is clearly visible. Some scan anomalies are also visible as thick black lines in Figures $2 \mathrm{~b}$ and $2 \mathrm{c}$ : this further informed our choice of region for high magnification SEM image acquisition.

Typical high magnification $(9,000 \times)$ SEM images are shown in Figure 3. The regions were nanoablated by raster scanning at $\lambda=760 \mathrm{~nm}$ with total pulse energy increasing from 0.7 to $4.3 \mu \mathrm{J}$, corresponding to applied average power of 8 to $51 \mathrm{~mW}$. Similar data were obtained for each wavelength used over the operating range of the laser source.

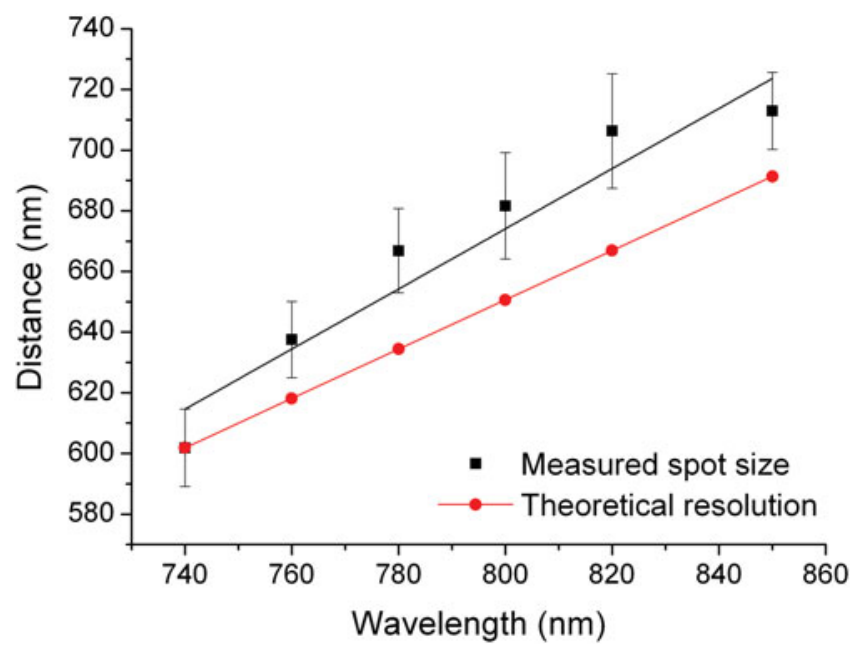

Figure 5. Lateral spot size determined by analysis of SEM images of nanoablated raster scans (squares). The lateral spot size values come from the gradient of the best fit lines associated with experimental measurements presented in Figure 4. On the same scale is the theoretical lateral resolution given by equation (1) for the same numerical aperture and wavelengths used (circles). We observe a good agreement between experimental and theoretical data, with less than a $10 \%$ deviation from the values predicted using equation (1).

It is evident from the high magnification images shown in Figure 3 that the nanoablated zone at low pulse energies is less homogeneous than at high pulse energies. We attribute this to nonablated aluminum remaining within the track. This contributed toward an increase in the error of the line widths obtained following nanoablation with low pulse energies, and therefore a similar increase in the error of the calculated lateral spot size.

\section{Image Analysis}

The mean nanoablated line width measured from the high magnification SEM images at $1 / e^{2}$ intensity was measured with increasing power for all wavelengths used in the nanoablation process, for $n=20$ measurements per image. The results were consistent with a linear increase in nanoablated line width with increasing average power.

To obtain a value for the focused beam radius, the line width data obtained from SEM image analysis were squared and plotted against the natural log of the incident pulse energy for each wavelength used in the nanoablation process. This is presented in Figure 4, where the measurements are quoted with standard deviation in the mean. With the gradient of the line equivalent to $2 w_{0}^{2}$, the value of the beam radius can then be determined from the best fit.

\section{Spot Size Measurement}

The values of beam radius obtained from the gradients of the best fit line in Figure 4 are presented for each wavelength in Figure 5. For comparison, the theoretical resolution calculated from equation (1) for the $20 \times / 0.75$ N.A. dry objective lens used during the nanoablation process at each wavelength is also shown in Figure 5. We observe a fair 
agreement between experimental and theoretical data, with less than a $10 \%$ deviation from the values predicted using equation (1).

\section{Discussion}

We have proposed and tested a novel measurement technique for use with multiphoton LSMs. The effectiveness of this technique is proven in Figure 5 where a reasonable agreement was found between our measurements and the theoretical resolution according the Abbe-Rayleigh diffraction limit (Abbe, 1884; Ditchburn, 1991). Our method offers improved accuracy because the scan line is swept rapidly, thus eliminating the effects of vibration at subkilohertz frequencies, which often limits the precision of measurements with fluorescent beads or gold nanoparticles. However, the main advantage comes from the use the high-resolution SEM to measure the LSM beam properties with $20 \mathrm{~nm}$ resolution. This allows for nanoablated line widths to be measured more accurately and, assuming Gaussian optics, a beam radius can be determined. It would be interesting to perform the nanoablation test with objectives within their design range of wavelengths, rather than those optimized for visible radiation but subsequently selected for high infrared transmission.

The wavelength effects observed in Figure 5 indicate that the resolution of the system is lower than theoretically expected at longer wavelengths. This is likely this is due to underfilling the back focal plane of the objective lens, which reduces the lateral resolution of the microscope (Muller et al., 2003). It would be desirable to repeat these measurements at the shorter wavelengths used for confocal laser scanning microscopy, but the commonly used lasers at these shorter wavelengths are continuous wave emitters with low average power, which we have found to be insufficient to perform nanoablation of aluminum. However, although other materials that we have tried, including gold, have proved much more difficult to nanoablate, alternative materials with low damage thresholds may be appropriate for similar measurement of confocal LSMs. We suggest that tin and zinc may have sufficiently low melting temperatures to be suitable for lower power ablation studies. In view of renewed interest in regenerative amplifier lasers for multiphoton microscopy (Mittman et al., 2011; Wang et al., 2011), we suggest that the high pulse energies available from these systems may be suitable with the materials and methods used here (Liang et al., 2011).

Although it was not our primary objective, we believe that this method may well prove useful also in evaluating LSMs because it provides a permanent record of precise scan parameters such as line spacing, local velocity variations, the jitter due to bearing error in galvanometer-driven mirrors, errors such as bouncing at the end of each scan, or flyback errors. It is particularly useful that the tests can be carried out in the LSM without removing the scanning mechanism, as, for example, if deterioration in mechanical performance of the galvo drive mechanism over long peri- ods of use is suspected. We also used dry objective lenses in our study, and we see no reason why the same method could not apply for glycerol, oil, or water immersion objective lenses, although some consideration for the compatibility of the specimen to be ablated with the immersion fluid may be required.

\section{CONCLUSION}

We have reported a novel approach to the measurement of the focal spot in laser scanning microscopy, which can be performed at laser powers currently used in biomedical microscopy. The results are precise and give absolute values broadly as expected from elementary diffraction theory. This approach is likely to be of value in the search for improved optical performance in the near-infrared. We suggest that this technique may also prove of value in servicing and developing laser scanning instruments.

\section{ACKNOWLEDGMENTS}

This work was funded by the Engineering and Physical Sciences Research Council (EP/I006826/1). We thank L. Tetley (University of Glasgow) and J. Skepper (University of Cambridge) for advice and help with SEM.

\section{REFERENCES}

Авве, E. (1884). A note on the proper definition of the amplifying power of a lens system. J Royal Microsc Soc 4, 348-351.

Amos, W. \& White, J. (1987). Use of confocal imaging in the study of biological structures. Appl Opt 26, 3239-3243.

Betzig, E., Patterson, G.H., Sougrat, R., Lindwasser, O.W., Olenych, S., Bonifacino, J.S., Davidson, M.W., LippincottSchwarz, J. \& Hess, H.F. (2006). Imaging intracellular fluorescent proteins at nanometre resolution. Science 313, 1642-1645.

Cox, G. \& Sheppard, C.J.R. (2004). Practical limits of resolution in confocal and non-linear microscopy. Microsc Res Tech 63, $18-22$.

Denk, W., Strickler, J.H. \& Webb, W.W. (1990). Two-photon laser scanning fluorescence microscopy. Science 248, 73.

Ditchburn, R.W. (1991). Light. New York: Dover Publications Inc.

Gordon, M.P., Ha, T. \& Selvin, P.R. (2004). Single-molecule high-resolution imaging with photobleaching. PNAS 101, 6462-6465.

Hell, S.W. \& Wichmann, J. (1994). Breaking the diffraction resolution limit by stimulated emission: Stimulated-emissiondepletion fluorescence microscopy. Opt Lett 19, 780-782.

Inoué, S. (2006). Foundations of confocal scanned imaging in light microscopy. In Handbook of Biological Confocal Microscopy, Pawley, J. (Ed.), pp. 1-19. New York: Springer.

Jee, Y., Becker, M.F. \& Walser, R.M. (1988). Laser-induced damage on single-crystal metal surfaces. J Opt Soc Am B 5, 648-659.

Klar, T.A., Jakobs, S., Dyba, M., Egner, A. \& Hell, S.W. (2000). Fluorescence microscopy with diffraction barrier broken by stimulated emission. PNAS 18, 8206-8210.

Liang, F., Vallee, R., Gingras, D. \& Chin, S.L. (2011). Role of ablation and incubation processes on surface nanograting formation. Opt Mat Exp 1, 1244-1250. 
Liv, J.M. (1982). Simple technique for measurements of pulsed Gaussian-beam spot sizes. Opt Lett 7, 196-198.

Liu, X., Du, D. \& Mourou, G. (1997). Laser ablation and micromachining with ultrashort laser pulses. IEEE J Quantum Elec 33, 1706-1716.

Mittman, W., Wallace, D.J., Czubayko, U., Herb, J.T., Schaefer, A.T., Looger, L.L., Denk, W. \& Kerr, J.N.D. (2011). Twophoton calcium imaging of evoked activity from L5 somatosenory neurons in vivo. Nat Neurosci 14, 1089-1093.

Muller, M., Schmidt, J.R., Mironov, S.L. \& Richter, D.W. (2003). Construction and performance of a custom-built twophoton laser scanning system. App Phys D 36, 1747-1757.

Oldenbourg, R., Terada, H., Tiberio, R. \& Inoué, S. (1993). Image sharpness and contrast transfer in coherent confocal microscopy. J Microsc 172, 31-39.

Sheppard, C. \& Kompfner, R. (1978). Resonant scanning optical microscope. App Opt 17, 2879-2882.
Siegman, A.E., Nemes, G. \& Serna, J. (1998). How to (maybe) measure laser beam quality. In DPSS (Diode Pumped Solid State Laser): Applications and Issues, Dowley, M. (Ed.), p. MQ1. Washington, DC: Optical Society of America.

Tan, B., Dalili, A. \& Venkatakrishnan, K. (2008). High repetition rate femtosecond laser nano-machining of thin films. Appl Phys A 95, 537-545.

Turner, G.L.E \& Bradbury, S. (1966). An electron microscopical examination of Norbert's finest test-plate of twenty bands. $J$ Royal Microsc Soc 85, 435-447.

Wang, C., Qiao, L., He, F., Cheng, Y. \& Xu, Z. (2011). Extension of imaging depth in two-photon fluorescence microscopy using a long-wavelength high-pulse-energy femtosecond laser source. J Microsc 243, 179-183.

ZuCKer, R.M. (2006). Evaluation of confocal microscopy system performance. Methods Mol Biol 319, 77-135. 